\title{
Teaching Video NeuroImages: Palatal myoclonus in leukodystrophies
}

\section{A clinical sign orienting to Alexander disease}

Javier Martinez-Poles, MD, José Bernardo Escribano-Paredes, MD, Sebastián García-Madrona, MD, Velina Nedkova-Hristova, MD, and Adriano Jiménez-Escrig, MD, PhD

Neurology ${ }^{\circledR}$ 2020;94:e230-e231. doi:10.1212/WNL.0000000000008795

Figure Brain MRI
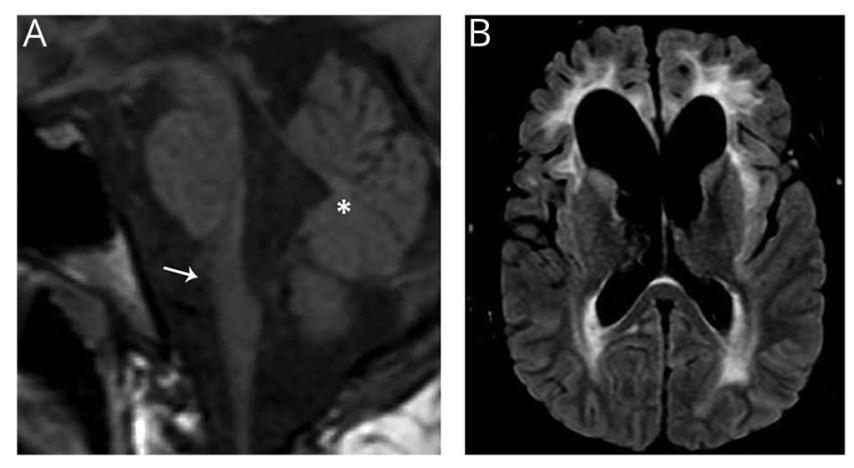

(A) Sagittal T1 MRI brain shows atrophy of brainstem (arrow) and cerebellum (asterisk). (B) Axial fluidattenuated inversion recovery sequence shows extensive white matter hyperintensities.

A 22-year-old man with Alexander disease type II diagnosed by a compatible MRI with bilateral white matter hyperintensities and brainstem atrophy (figure) and mutation c.236G $>\mathrm{A}$ (p.Arg79His) in the GFAP gene presented with recent onset continuous palatal myoclonus without ear clicking (video 1).

Palatal myoclonus is caused by a lesion in the triangle of Guillain-Mollaret (formed by dentate nucleus, red nucleus, and inferior olivary nucleus) and associated with hypertrophic olivary degeneration. ${ }^{1}$ As Alexander disease is a leukodystrophy that predominantly affects the brainstem, palatal myoclonus can be a useful sign to distinguish it from other leukodystrophies. ${ }^{2}$

\section{Study funding}

No targeted funding reported.

\section{Disclosure}

The authors report no disclosures relevant to the manuscript. Go to Neurology.org/N for full disclosures. 


\section{Appendix Authors}

\begin{tabular}{|c|c|c|c|}
\hline Name & Location & Role & Contribution \\
\hline $\begin{array}{l}\text { Javier } \\
\text { Martinez- } \\
\text { Poles, MD }\end{array}$ & $\begin{array}{l}\text { Neurology } \\
\text { Department, Ramón y } \\
\text { Cajal University } \\
\text { Hospital, Madrid, } \\
\text { Spain }\end{array}$ & Author & $\begin{array}{l}\text { Drafted the } \\
\text { manuscript for } \\
\text { intellectual content, } \\
\text { major role in the } \\
\text { acquisition of data }\end{array}$ \\
\hline $\begin{array}{l}\text { José } \\
\text { Bernardo } \\
\text { Escribano- } \\
\text { Paredes, } \\
\text { MD }\end{array}$ & $\begin{array}{l}\text { Neurology } \\
\text { Department, Ramón y } \\
\text { Cajal University } \\
\text { Hospital, Madrid, } \\
\text { Spain }\end{array}$ & Author & $\begin{array}{l}\text { Revised the } \\
\text { manuscript for } \\
\text { intellectual content }\end{array}$ \\
\hline $\begin{array}{l}\text { Sebastián } \\
\text { Garcia- } \\
\text { Madrona, } \\
\text { MD }\end{array}$ & $\begin{array}{l}\text { Neurology } \\
\text { Department, Ramón y } \\
\text { Cajal University } \\
\text { Hospital, Madrid, } \\
\text { Spain }\end{array}$ & Author & $\begin{array}{l}\text { Revised the } \\
\text { manuscript for } \\
\text { intellectual content }\end{array}$ \\
\hline
\end{tabular}

Appendix (continued)

\begin{tabular}{|c|c|c|c|}
\hline Name & Location & Role & Contribution \\
\hline $\begin{array}{l}\text { Velina } \\
\text { Nedkova- } \\
\text { Hristova, } \\
\text { MD }\end{array}$ & $\begin{array}{l}\text { Neurology } \\
\text { Department, Ramón y } \\
\text { Cajal University } \\
\text { Hospital, Madrid, } \\
\text { Spain }\end{array}$ & Author & $\begin{array}{l}\text { Revised the } \\
\text { manuscript for } \\
\text { intellectual content }\end{array}$ \\
\hline $\begin{array}{l}\text { Adriano } \\
\text { Jiménez- } \\
\text { Escrig, MD, } \\
\text { PhD }\end{array}$ & $\begin{array}{l}\text { Neurology } \\
\text { Department, Ramón y } \\
\text { Cajal University } \\
\text { Hospital, Madrid, } \\
\text { Spain }\end{array}$ & Author & $\begin{array}{l}\text { Major role in the } \\
\text { acquisition of data, } \\
\text { revised the } \\
\text { manuscript for } \\
\text { intellectual content }\end{array}$ \\
\hline
\end{tabular}

\section{References}

1. Pearce JMS. Palatal myoclonus (syn palatal tremor). Eur Neurol 2008;60:312-315.

2. Howard KL, Hall DA, Moon M, Agarwal P, Newman E, Brenner M. Adult-onset Alexander disease with progressive ataxia and palatal tremor. Mov Disord 2008;23:118-122. 


\section{Neurology}

\section{Teaching Video NeuroImages: Palatal myoclonus in leukodystrophies: A clinical sign orienting to Alexander disease}

Javier Martinez-Poles, José Bernardo Escribano-Paredes, Sebastián García-Madrona, et al. Neurology 2020;94;e230-e231

DOI 10.1212/WNL.0000000000008795

\section{This information is current as of January 13, 2020}

\section{Updated Information \&} Services

References

Subspecialty Collections

Permissions \& Licensing

Reprints including high resolution figures, can be found at: http://n.neurology.org/content/94/2/e230.full

This article cites 2 articles, 0 of which you can access for free at: http://n.neurology.org/content/94/2/e230.full\#ref-list-1

This article, along with others on similar topics, appears in the following collection(s):

Clinical neurology examination

http://n.neurology.org/cgi/collection/clinical_neurology_examination Leukodystrophies

http://n.neurology.org/cgi/collection/leukodystrophies Myoclonus

http://n.neurology.org/cgi/collection/myoclonus

Information about reproducing this article in parts (figures,tables) or in its entirety can be found online at:

http://www.neurology.org/about/about_the_journal\#permissions

Information about ordering reprints can be found online:

http://n.neurology.org/subscribers/advertise

Neurology ${ }^{\circledR}$ is the official journal of the American Academy of Neurology. Published continuously since 1951, it is now a weekly with 48 issues per year. Copyright () 2020 American Academy of Neurology. All rights reserved. Print ISSN: 0028-3878. Online ISSN: 1526-632X.

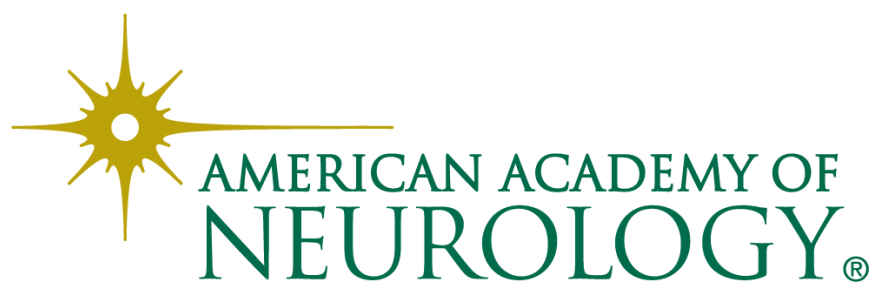

